Supplement of Atmos. Chem. Phys., 16, 8939-8962, 2016

http://www.atmos-chem-phys.net/16/8939/2016/

doi:10.5194/acp-16-8939-2016-supplement

(C) Author(s) 2016. CC Attribution 3.0 License.

(c) (i)

Supplement of

\title{
Global combustion sources of organic aerosols: model comparison with 84 AMS factor-analysis data sets
}

\author{
Alexandra P. Tsimpidi et al. \\ Correspondence to: Alexandra P. Tsimpidi (a.tsimpidi@mpic.de)
}

The copyright of individual parts of the supplement might differ from the CC-BY 3.0 licence. 
Table S1. Statistical evaluation of EMAC simulated inorganic gas and aerosol concentrations against monthly average observations from North America during 2001-2010. The units are in ppb for gas and in $\mu \mathrm{g} \mathrm{m}^{-3}$ for aerosol species.

EPA Network for gas-phase species and IMPROVE Network for aerosols

\begin{tabular}{lccccc} 
Metric & $\mathbf{S O}_{2}$ & $\mathbf{N O}_{2}$ & $\mathbf{S O}_{4}{ }^{2-}$ & $\mathbf{N O}_{3}{ }^{-}$ & $\mathbf{N H}_{4}{ }^{+}$ \\
\hline Observed & 3.35 & 12.07 & 1.50 & 0.71 & 1.12 \\
Simulated & 1.89 & 4.22 & 0.83 & 0.52 & 0.82 \\
MAGE & 2.03 & 8.07 & 1.12 & 0.50 & 0.54 \\
MB & -1.46 & -7.85 & -0.67 & -0.19 & -0.30 \\
NME (\%) & 61 & 67 & 75 & 70 & 49 \\
NMB (\%) & -44 & -65 & -44 & -26 & -27 \\
RMSE & 4.27 & 10.36 & 1.76 & 1.06 & 0.65 \\
$\begin{array}{l}\text { Number of } \\
\text { comparisons }\end{array}$ & 53094 & 46250 & 17129 & 39056 & 481 \\
\hline
\end{tabular}


Table S2. Statistical evaluation of EMAC simulated inorganic gas and aerosol concentrations against monthly average observations from Europe during 2001-2010. The units are in $\mu \mathrm{g} \mathrm{m}^{-3}$ for both gas and aerosol species.

EMEP Network

\begin{tabular}{lccccc} 
Metric & $\mathbf{S O}_{2}$ & $\mathbf{N O}_{2}$ & $\mathbf{S O}_{4}{ }^{2-}$ & $\mathbf{N O}_{3}{ }^{-}$ & $\mathbf{N H}_{4}{ }^{+}$ \\
\hline Observed & 1.54 & 6.98 & 1.35 & 1.12 & 0.83 \\
Simulated & 2.93 & 7.28 & 1.00 & 1.34 & 0.76 \\
MAGE & 1.90 & 3.58 & 0.82 & 1.17 & 0.48 \\
MB & 1.39 & 0.3 & -0.35 & 0.22 & -0.07 \\
NME (\%) & 123 & 51 & 61 & 105 & 58 \\
NMB (\%) & 90 & 4 & -25 & 19 & -8 \\
RMSE & 2.97 & 7.18 & 1.27 & 2.14 & 0.74 \\
$\begin{array}{l}\text { Number of } \\
\text { comparisons }\end{array}$ & 9130 & 8626 & 16707 & 9307 & 7787 \\
\hline
\end{tabular}


Table S3. Statistical evaluation of EMAC simulated inorganic gas and aerosol concentrations against monthly average observations from Eastern Asia during 20012010. The units are in ppb for gas and in $\mu \mathrm{g} \mathrm{m}^{-3}$ for aerosol species.

\section{EANET Network}

\begin{tabular}{lccccc} 
Metric & $\mathbf{S O}_{2}$ & $\mathbf{N O}_{2}$ & $\mathbf{S O}_{\mathbf{4}}{ }^{2-}$ & $\mathbf{N O}_{\mathbf{3}}{ }^{-}$ & $\mathbf{N H}_{4}{ }^{+}$ \\
\hline Observed & 2.80 & 14.40 & 3.78 & 1.13 & 1.05 \\
Simulated & 1.74 & 2.07 & 1.07 & 0.75 & 0.67 \\
MAGE & 1.90 & 12.59 & 2.84 & 0.93 & 0.68 \\
MB & -1.06 & -12.33 & -2.71 & -0.38 & -0.38 \\
NME (\%) & 68 & 87 & 75 & 82 & 64 \\
NMB (\%) & -38 & -86 & -72 & -33 & -36 \\
RMSE & 3.39 & 16.94 & 5.17 & 2.01 & 1.74 \\
$\begin{array}{l}\text { Number of } \\
\text { comparisons }\end{array}$ & 7076 & 926 & 2965 & 2862 & 2897 \\
\hline
\end{tabular}


Table S4. Secondary organic aerosol mass yield ${ }^{1}$ parameters

\begin{tabular}{ccccc}
\hline & \multicolumn{5}{c}{$\boldsymbol{C}^{*} \boldsymbol{i n} \boldsymbol{\mu} \boldsymbol{g} \boldsymbol{m}^{-3} \boldsymbol{a t} \mathbf{2 9 8 K}$} \\
\cline { 2 - 5 } VOC Species & 1 & 10 & $10^{2}$ & $10^{3}$ \\
\hline Benzene, Toluene & 0.003 & 0.165 & 0.300 & 0.435 \\
Trimetyl Benzenes, Xylene, and other Aromatics & 0.002 & 0.195 & 0.300 & 0.435 \\
Pentanes & 0.000 & 0.038 & 0.000 & 0.000 \\
Hexanes, other higher Alkenes & 0.000 & 0.150 & 0.000 & 0.000 \\
Propene & 0.001 & 0.005 & 0.038 & 0.150 \\
Other Alkenes & 0.003 & 0.026 & 0.083 & 0.270 \\
Isoprene & 0.009 & 0.030 & 0.015 & 0.000 \\
Monoterpenes & 0.107 & 0.092 & 0.359 & 0.600 \\
\hline
\end{tabular}

${ }^{1}$ The SOA yields are based on an assumed particle density of $1.5 \mathrm{~g} \mathrm{~cm}^{-3}$. 


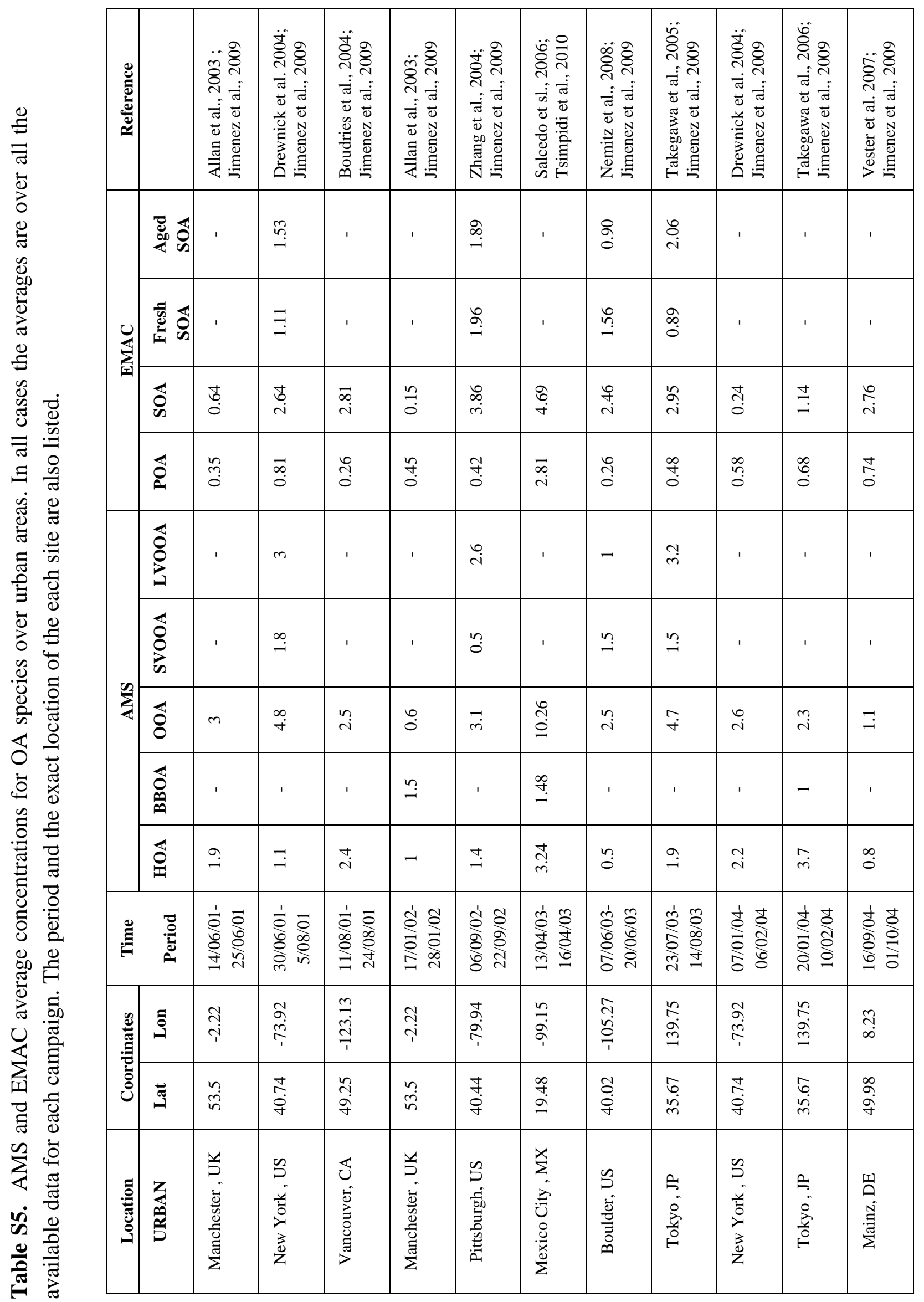




\begin{tabular}{|c|c|c|c|c|c|c|c|c|c|c|c|c|c|}
\hline 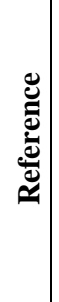 & & 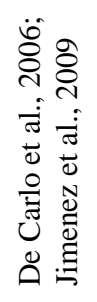 & 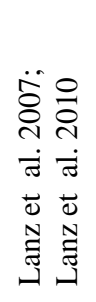 & 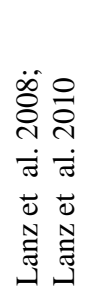 & 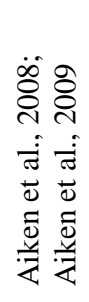 & 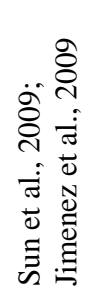 & 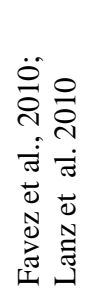 & 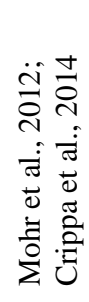 & 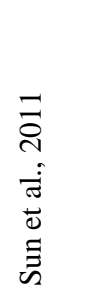 & 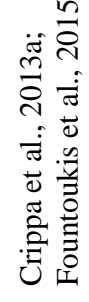 & 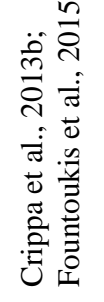 & 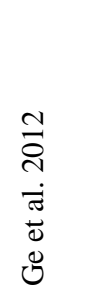 & 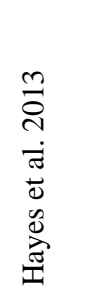 \\
\hline \multirow{4}{*}{$\sum_{i=1}^{0}$} & 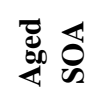 & $\frac{5}{6}$ & $\stackrel{\infty}{\infty}$ & ' & ' & $\stackrel{+}{\stackrel{n}{r}}$ & & $\stackrel{\infty}{\stackrel{0}{0}}$ & $\stackrel{\sim}{\infty}$ & & ' & I & 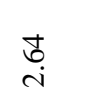 \\
\hline & 苞 & $\stackrel{\Re}{\stackrel{\leftrightarrow}{+}}$ & $\stackrel{n}{n}$ & ' & ' & $\stackrel{n}{n}$ & & $\underset{0}{F}$ & $\stackrel{n}{=}$ & & ' & ' & $\underset{i}{\stackrel{8}{0}}$ \\
\hline & ర్ర & $\hat{\varrho}$ & $\bar{m}$ & 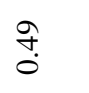 & $\underset{\dot{+}}{\vec{\sigma}}$ & $=$ & $\stackrel{?}{\circ}$ & $\stackrel{9}{\rightleftarrows}$ & 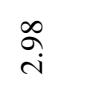 & $\stackrel{\infty}{n}$ & đ̃ & $\underset{0}{\stackrel{g}{0}}$ & $\underset{+}{\stackrel{+}{+}}$ \\
\hline & ఏ & $\stackrel{0}{=}$ & $n$ & $\stackrel{\infty}{\stackrel{\infty}{\circ}}$ & $\stackrel{m}{i}$ & $\stackrel{n}{\dot{m}}$ & $\stackrel{?}{\stackrel{f}{-}}$ & $\stackrel{?}{0}$ & $\stackrel{\infty}{\circ}$ & $\stackrel{\circ}{\circ}$ & $\stackrel{+}{-}$ & $\tilde{\jmath}$ & $\hat{o}$ \\
\hline \multirow{5}{*}{$\sum_{4}^{\infty}$} & $\begin{array}{l}8 \\
8 \\
0 \\
3\end{array}$ & $\stackrel{\infty}{i}$ & $\begin{array}{l}\tilde{\infty} \\
i\end{array}$ & ' & ' & $\stackrel{3}{\Omega}$ & 1 & $\stackrel{\infty}{i}$ & $\stackrel{\Omega}{\Omega}$ & ' & ' & ' & 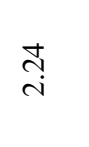 \\
\hline & 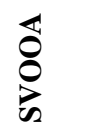 & $\stackrel{\sim}{+}$ & $\stackrel{f}{\stackrel{f}{*}}$ & ' & ' & $\stackrel{?}{+}$ & 1 & $\underset{\sim}{\mathbb{S}}$ & $\underset{i}{\Delta}$ & ' & ' & ' & 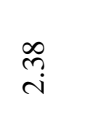 \\
\hline & ठ্ & $r$ & $\overrightarrow{\tilde{\jmath}}$ & $\stackrel{n}{n}$ & $\stackrel{?}{?}$ & $\stackrel{0}{\dot{0}}$ & 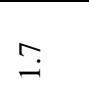 & $\underset{+}{\tilde{\sigma}}$ & $\underset{f}{0}$ & 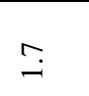 & $\stackrel{\sim}{r}$ & $\stackrel{\vec{r}}{\dot{r}}$ & $\begin{array}{l}\tilde{\sigma} \\
+\end{array}$ \\
\hline & $\begin{array}{l}\overleftarrow{0} \\
\stackrel{0}{*}\end{array}$ & $\stackrel{0}{0}$ & : & $\stackrel{n}{\stackrel{n}{\leftrightarrows}}$ & $\tilde{i}$ & ' & $\stackrel{n}{n}$ & : & , & , & , & $\stackrel{\text { }}{\stackrel{\overbrace{}}{I}}$ & t \\
\hline & 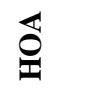 & $\stackrel{\sim}{\longrightarrow}$ & $\stackrel{0}{+}$ & $\stackrel{?}{3}$ & $\stackrel{\circ}{+}$ & $\stackrel{n}{=}$ & $\stackrel{+}{0}$ & $\hat{a}$ & $\bar{\sigma}$ & $\hat{0}$ & $\hat{i}$ & 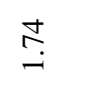 & $\underset{\infty}{+}$ \\
\hline$\stackrel{\mathscr{\Xi}}{\Xi}$ & 苞 & 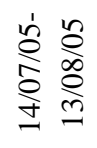 & 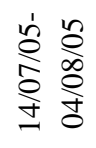 & 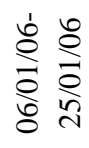 & 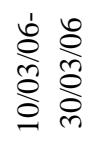 & 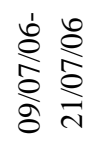 & 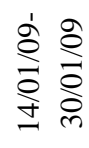 & 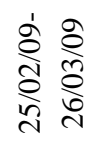 & 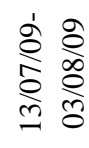 & 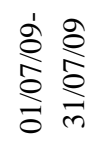 & 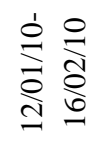 & 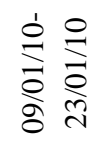 & 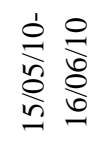 \\
\hline 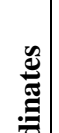 & $\Xi$ & 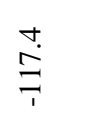 & $\begin{array}{l}n \\
\infty\end{array}$ & $\ddot{\infty}$ & $\frac{n}{a}$ & $\stackrel{0}{\stackrel{0}{=}}$ & $\stackrel{\overbrace{}}{i}$ & $\underset{i}{i}$ & $\stackrel{\sigma}{\stackrel{n}{r}}$ & 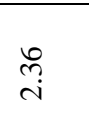 & గొ & $\stackrel{\infty}{\stackrel{\infty}{\sigma}}$ & 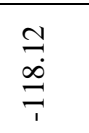 \\
\hline نे & 疍 & $\begin{array}{l}n \\
\stackrel{n}{m}\end{array}$ & $\underset{\dot{f}}{\stackrel{+}{\dot{f}}}$ & $\stackrel{\stackrel{+}{\dot{q}}}{\text {. }}$ & $\stackrel{\infty}{\stackrel{0}{9}}$ & 字 & $\stackrel{\infty}{\dot{b}}$ & $\stackrel{\oplus}{\vec{\forall}}$ & $\stackrel{+}{\stackrel{g}{q}}$ & $\begin{array}{l}\infty \\
\infty \\
\infty \\
\infty\end{array}$ & $\begin{array}{l}\infty \\
\infty \\
\dot{\infty}\end{array}$ & $\begin{array}{l}\vec{\infty} \\
\dot{\delta}\end{array}$ & $\underset{\dot{m}}{\stackrel{+}{\dot{H}}}$ \\
\hline 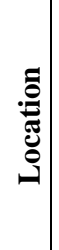 & $\begin{array}{l}\frac{z}{4} \\
\frac{0}{5} \\
\frac{3}{5}\end{array}$ & 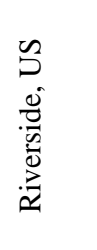 & 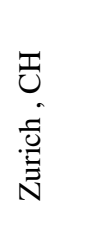 & 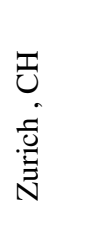 & 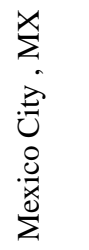 & $\begin{array}{l}Z \\
\text { Z } \\
\vdots 0 \\
\vdots \overline{\overrightarrow{0}} \\
\infty\end{array}$ & 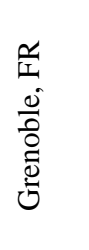 & 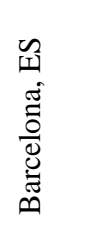 & $\begin{array}{l}n \\
0 \\
0 \\
0 \\
0 \\
z \\
z \\
z\end{array}$ & 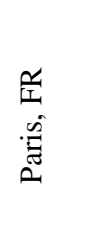 & 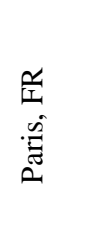 & 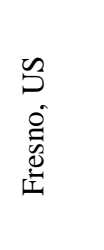 & 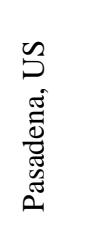 \\
\hline
\end{tabular}




\begin{tabular}{|c|c|c|c|c|c|c|c|c|c|c|c|c|c|c|c|c|}
\hline$\approx$ & & 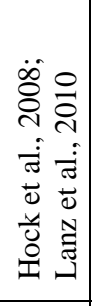 & 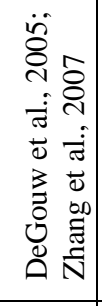 & 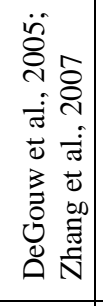 & 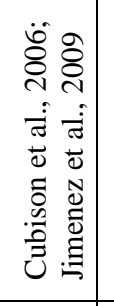 & 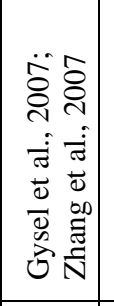 & 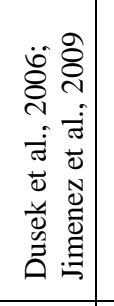 & 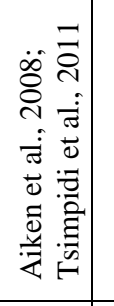 & 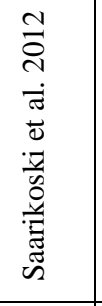 & 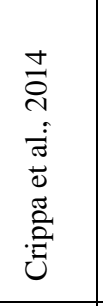 & 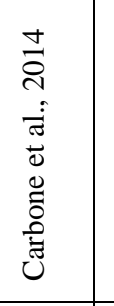 & 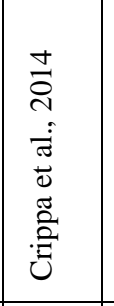 & 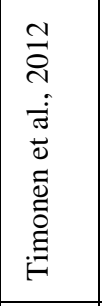 & 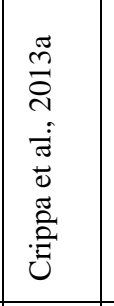 & 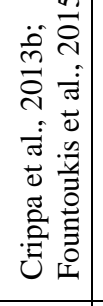 & 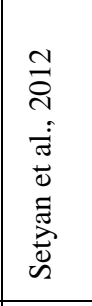 \\
\hline & $\underset{\mathrm{d}}{\mathrm{d}} \mathrm{d}$ & & & & 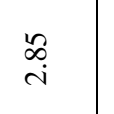 & & $\stackrel{0}{\rightarrow}$ & & ' & $\stackrel{8}{0}$ & $\stackrel{\infty}{0}$ & 8 & $\underset{\infty}{\infty}$ & 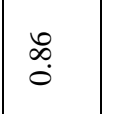 & & 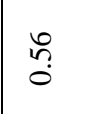 \\
\hline & $\overline{\underline{s}}$ & . & ' & & $\stackrel{\infty}{\circ}$ & & $\stackrel{\mathscr{m}}{\dddot{m}}$ & & ' & $\stackrel{5}{0}$ & ?ृ & F. & $\stackrel{\circ}{8}$ & $\stackrel{\circ}{\circ}$ & & $\stackrel{\overbrace{}}{9}$ \\
\hline & $\stackrel{1}{8}$ & $\overline{\text { i }}$ & 号 & $\stackrel{\leftrightarrow}{\Im}$ & : & 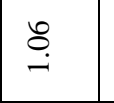 & $\stackrel{\infty}{i}$ & 帝 & $\stackrel{ल}{3}$ & $\stackrel{m}{0}$ & f. & $\stackrel{9}{=}$ & $\stackrel{M}{=}$ & 声 & तु & $\stackrel{\infty}{\leftrightarrow}$ \\
\hline & $\stackrel{d}{d}$ & fo & $\begin{array}{l}\infty \\
\infty \\
\infty \\
\infty\end{array}$ & ্ֻ & $\stackrel{8}{\circ}$ & ầ & $\stackrel{8}{8}$ & $a$ & $\stackrel{\infty}{a}$ & $\stackrel{\circ}{\circ}$ & $\stackrel{8}{-}$ & $\frac{1}{8}$ & $n$ & bi & $\stackrel{\infty}{\rightarrow}$ & ฮู \\
\hline & 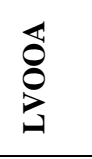 & , & & & \pm & & $q$ & & ' & ó & $\stackrel{ \pm}{ \pm}$ & $\vec{i}$ & $\stackrel{\rho}{I}$ & r̃ & & à \\
\hline & $\stackrel{\Delta}{0}$ & , & & & $\stackrel{ \pm}{0}$ & & $\stackrel{+}{\sim}$ & & ' & 0 & f & 0 & 苦 & 苛 & & $\stackrel{\text { I }}{2}$ \\
\hline$\frac{2}{4}$ & $\ddot{8}$ & 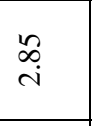 & $\stackrel{+t}{b}$ & $\stackrel{+}{+}$ & $\stackrel{\infty}{\longrightarrow}$ & $\because$ & $\stackrel{a}{\stackrel{2}{2}}$ & $\stackrel{+}{+}$ & $\stackrel{\leftrightarrow}{=}$ & م. & $\stackrel{\infty}{-\infty}$ & $\vec{i}$ & $\frac{m}{i}$ & $\stackrel{9}{\ni}$ & $\stackrel{m}{m}$ & $\stackrel{\overline{\mathrm{i}}}{\overline{\mathrm{s}}}$ \\
\hline & 总 & . & & & & & ' & & 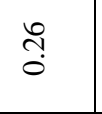 & & \%్ & तु & 8 & ' & & \\
\hline & $\overleftrightarrow{\Phi}$ & 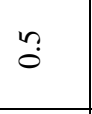 & $\stackrel{t}{0}$ & 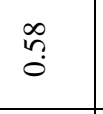 & $\stackrel{\infty}{\infty}$ & 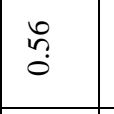 & $\stackrel{\circ}{\longrightarrow}$ & $\stackrel{3}{3}$ & 尺े & 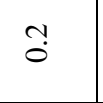 & f. & à & 志 & $\because$ & $\stackrel{+}{\mathrm{i}}$ & Iี \\
\hline & 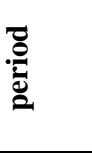 & 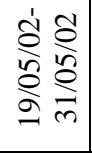 & 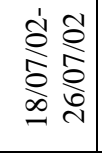 & 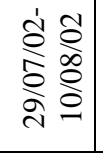 & 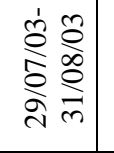 & 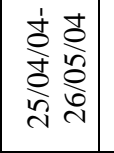 & 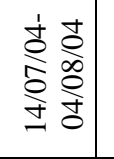 & 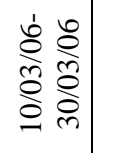 & 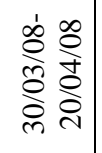 & 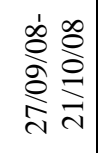 & 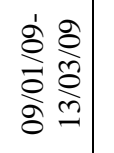 & 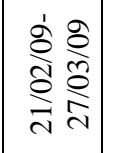 & 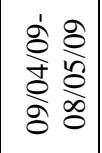 & 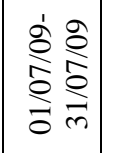 & 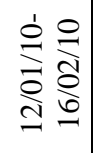 & 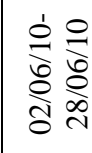 \\
\hline & $\Xi$ & $\stackrel{\odot}{=}$ & $\underset{P}{P}$ & $\underset{P}{P}$ & $\stackrel{+}{0}$ & $\exists$ & 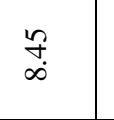 & 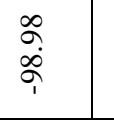 & $\stackrel{\text { }}{=}$ & ¿̊c & 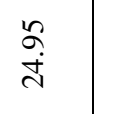 & गे & $\begin{array}{l}\text { 品 } \\
\text { do }\end{array}$ & $\vec{i}$ & $\bar{i}$ & $\frac{\stackrel{P}{\vec{I}}}{\mathrm{~T}}$ \\
\hline & $\vec{\Xi}$ & $\begin{array}{l}\infty \\
\stackrel{f}{f}\end{array}$ & 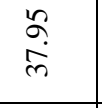 & 点 & $\stackrel{\Gamma}{n}$ & in & ָี & $\Phi$ & $\begin{array}{l}\text { 岁 } \\
\dot{f}\end{array}$ & बें & 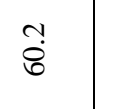 & 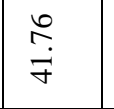 & हैं & 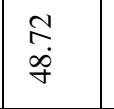 & 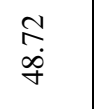 & \begin{tabular}{|l}
$\infty$ \\
$\infty$ \\
$\infty$ \\
$\infty$ \\
$\infty$
\end{tabular} \\
\hline & 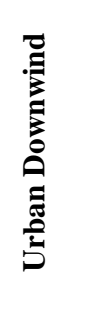 & 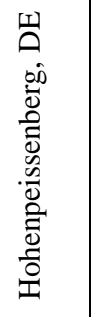 & 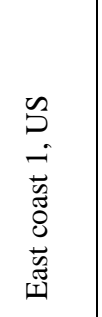 & 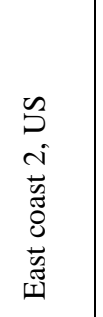 & 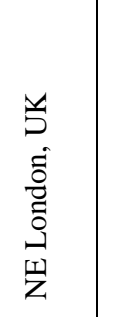 & 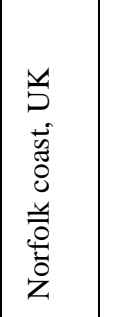 & 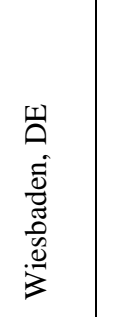 & 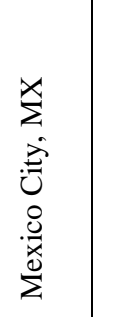 & 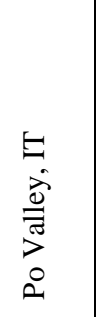 & 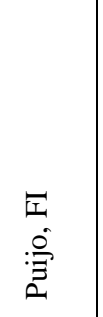 & 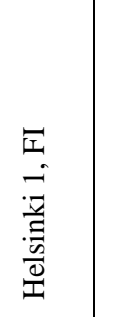 & 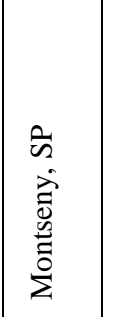 & 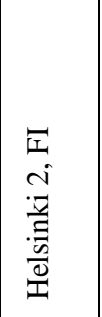 & 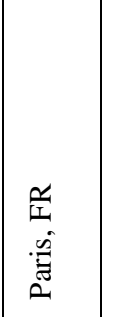 & 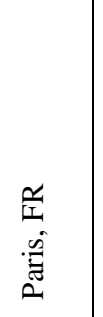 & $\begin{array}{l}3 \\
\frac{2}{8} \\
\frac{8}{8}\end{array}$ \\
\hline
\end{tabular}




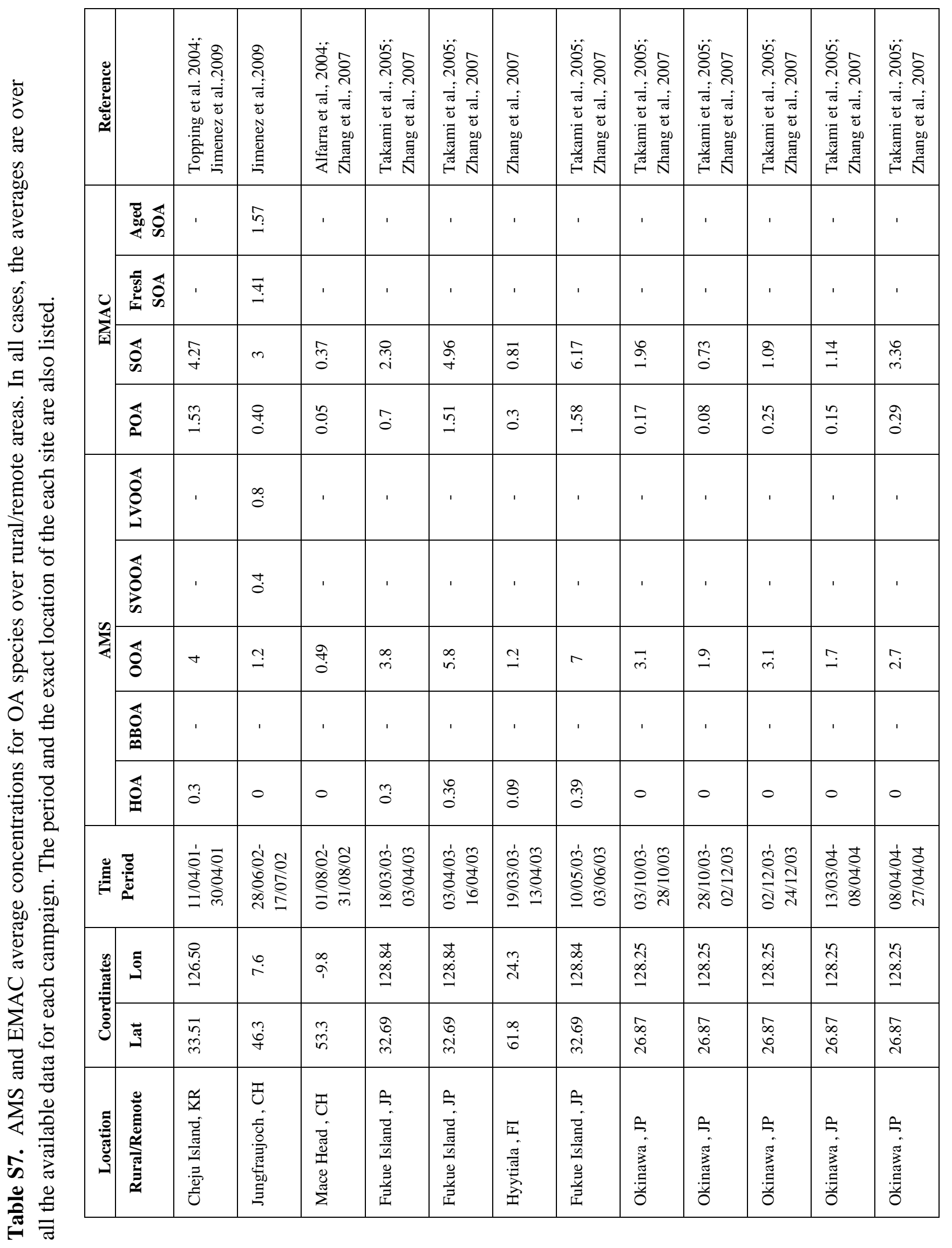




\begin{tabular}{|c|c|c|c|c|c|c|c|c|c|c|c|}
\hline |l: & 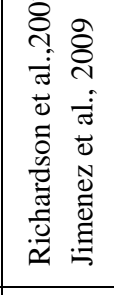 & 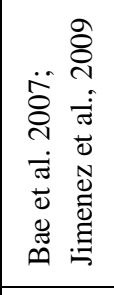 & 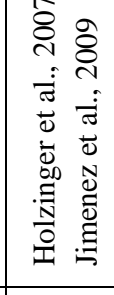 & 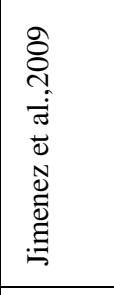 & 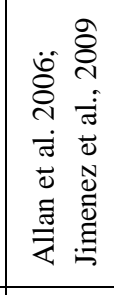 & 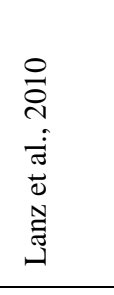 & 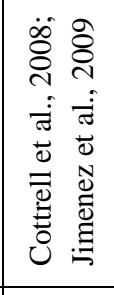 & 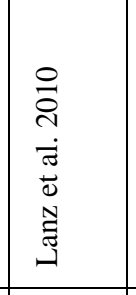 & 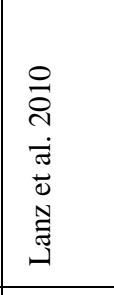 & 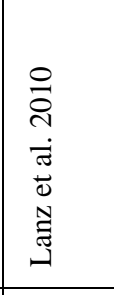 & 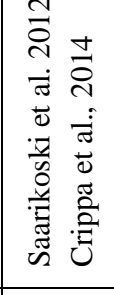 \\
\hline 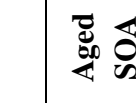 & & $\stackrel{\rho}{g}$ & 9 & $\stackrel{\mathscr{m}}{3}$ & 葵 & $\stackrel{0}{9}$ & $\vec{i}_{i}$ & $\stackrel{ \pm}{0}$ & & 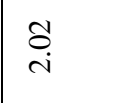 & : \\
\hline 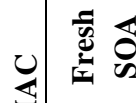 & & $\stackrel{9}{=}$ & gे & $\stackrel{\infty}{ \pm}$ & 声 & : & E & 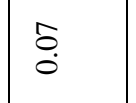 & & gे & 号 \\
\hline 雨荅 & $\infty$ & గై & $\stackrel{\Xi}{-}$ & $\overrightarrow{\vec{\Phi}}$ & 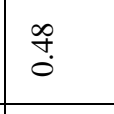 & בุ & $\underset{\infty}{\infty}$ & 공 & : & 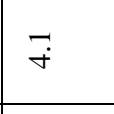 & $\stackrel{\widetilde{\Omega}}{2}$ \\
\hline$\overleftrightarrow{\varrho}$ & పे & m. & $\because$ & ه̊ & đุ & s & 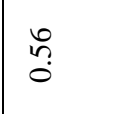 & g. & કิ & 苦 & 我 \\
\hline 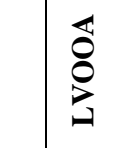 & & $\bar{i}$ & gे & $\stackrel{\infty}{\infty}$ & $\stackrel{0}{\circ}$ & 索 & 3 & 8 & & 它 & 9 \\
\hline ठ̊. & & $\bar{i}$ & $\stackrel{\circ}{\circ}$ & $a$ & $\stackrel{0}{0}$ & $\underset{\text { i }}{\tilde{i}}$ & iे & s. & & 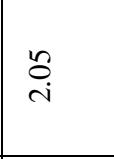 & $\stackrel{\leftrightarrow}{-}$ \\
\hline$\frac{\pi}{8}$ & 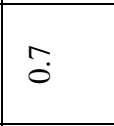 & 苟 & $\because$ & 9 & $\cong$ & ְ̊: & f & $\stackrel{\circ}{+}$ & $\overrightarrow{\dot{\alpha}}$ & 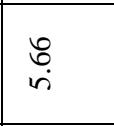 & స̊. \\
\hline 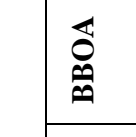 & & & , & ' & . & . & , & ' & $\exists$ & $=$ & 导 \\
\hline 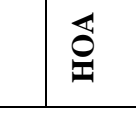 & İ & 3 & ב & $\overline{0}$ & 0 & gे & $=$ & 8 & $\vec{b}$ & 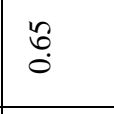 & 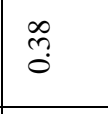 \\
\hline 总营 & $\begin{array}{l}1 \\
0 \\
0 \\
0 \\
0 \\
0 \\
0\end{array}$ & 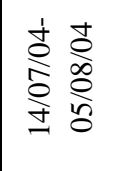 & 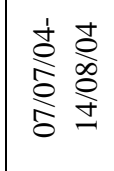 & 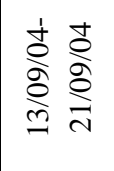 & 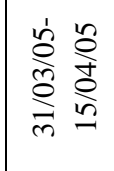 & 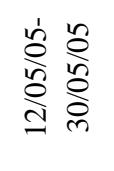 & 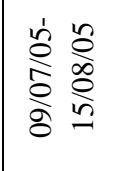 & 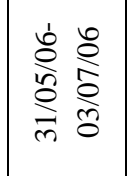 & ஓे & 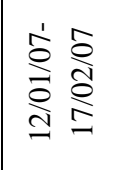 & 离 \\
\hline 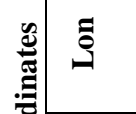 & $\mid \frac{\hat{\theta}}{\vec{\theta}}$ & $\stackrel{0}{i}$ & $\vec{b}$ & $\overrightarrow{\bar{s}}$ & $\stackrel{?}{\mathfrak{d}}$ & $\stackrel{\infty}{\sim}$ & $\mid \begin{array}{l}\text { à } \\
\text { ô. } \\
i\end{array}$ & o & t. & 蓝 & $\stackrel{\leftrightarrow}{\stackrel{\leftrightarrow}{=}}$ \\
\hline ठิ & $\mid \begin{array}{l}\frac{1}{8} \\
8 \\
q\end{array}$ & 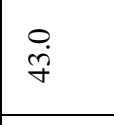 & 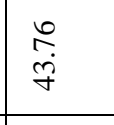 & 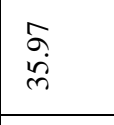 & $\underset{0}{\infty}$ & $\begin{array}{c}\text { 薃 } \\
\end{array}$ & $\begin{array}{l}\overline{7} \\
\overline{9}\end{array}$ & $\begin{array}{l}\infty \\
\dot{q} \\
\dot{y}\end{array}$ & 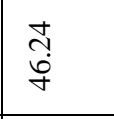 & $\begin{array}{l}\infty \\
\text { ơ } \\
\end{array}$ & \begin{tabular}{|l} 
\\
\hdashline \\
$q$ \\
\end{tabular} \\
\hline 童 & 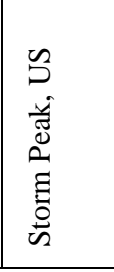 & 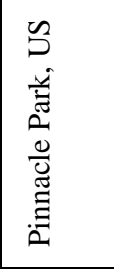 & 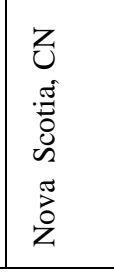 & 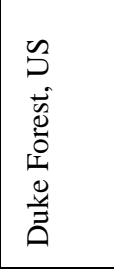 & 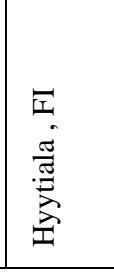 & 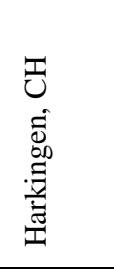 & 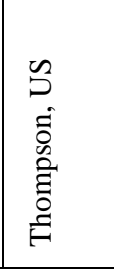 & 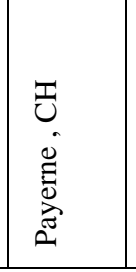 & 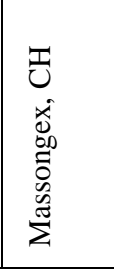 & 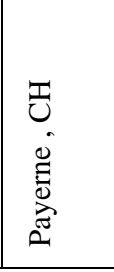 & 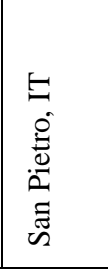 \\
\hline
\end{tabular}




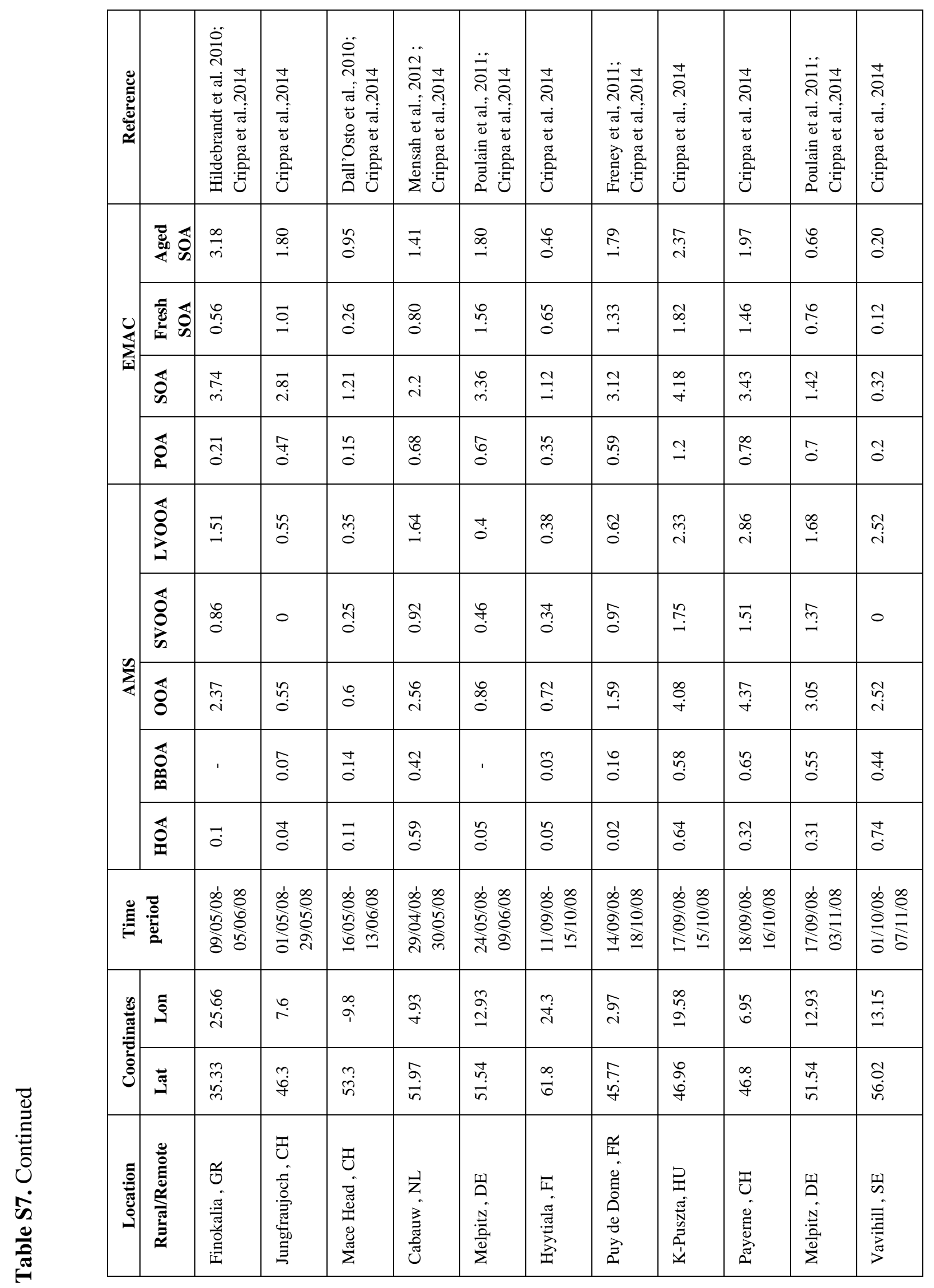




\begin{tabular}{|c|c|c|c|c|c|c|c|c|c|c|c|c|c|}
\hline 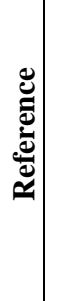 & & 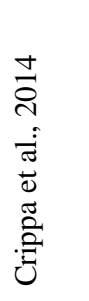 & 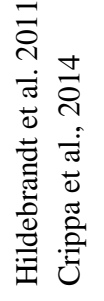 & 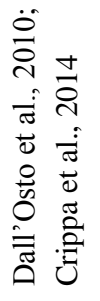 & 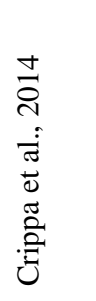 & 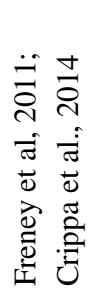 & 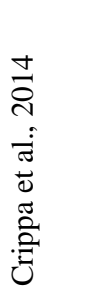 & 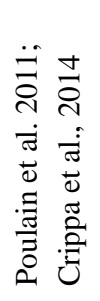 & 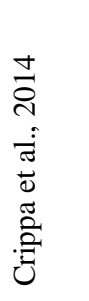 & 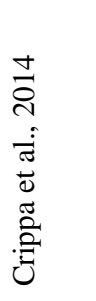 & 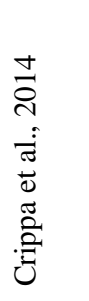 & 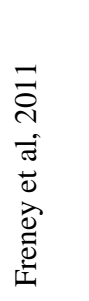 & 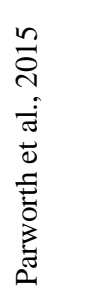 \\
\hline \multirow{4}{*}{$\sum_{x=1}^{0}$} & \begin{tabular}{l}
$\mathbb{S}_{4}$ \\
\hdashline
\end{tabular} & $\stackrel{\Xi}{\tilde{O}}$ & $\stackrel{ }{\stackrel{0}{0}}$ & $\stackrel{m}{0}$ & $\stackrel{\widetilde{n}}{0}$ & on & $\stackrel{5}{0}$ & 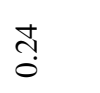 & $\stackrel{n}{\tilde{o}}$ & $\stackrel{\infty}{0}$ & $\stackrel{0}{0}$ & 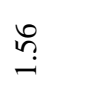 & ָे \\
\hline & 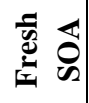 & $\stackrel{\circ}{\circ}$ & $\frac{n}{0}$ & $\stackrel{n}{0}$ & $\stackrel{0}{0}$ & $\stackrel{\infty}{\sim}$ & $\frac{1}{0}$ & $\frac{0}{0}$ & $\frac{9}{0}$ & $\stackrel{0}{\circ}$ & $\stackrel{8}{\circ}$ & $\stackrel{?}{\sim}$ & 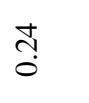 \\
\hline & 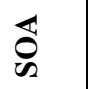 & 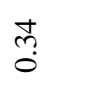 & ô & $\stackrel{\infty}{\circ}$ & $\stackrel{t}{0}$ & $\stackrel{n}{\mathfrak{0}}$ & तิ & $\stackrel{+}{0}$ & $\stackrel{+}{n}$ & $\stackrel{\infty}{\stackrel{\infty}{0}}$ & $\stackrel{\text { }}{3}$ & $\stackrel{\text { }}{\text { i }}$ & $\tilde{n}$ \\
\hline & 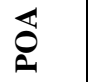 & $\tilde{\overbrace{}}$ & $\stackrel{\circ}{\circ}$ & $\stackrel{8}{\circ}$ & $\stackrel{?}{\stackrel{0}{0}}$ & $\hat{n}$ & $\stackrel{i}{0}$ & ले & $\hat{m}$ & $\stackrel{3}{0}$ & $\stackrel{n}{3}$ & ্ֻণ & $\overrightarrow{\widetilde{o}}$ \\
\hline \multirow{5}{*}{$\sum_{4}^{n}$} & $\begin{array}{l}\overleftrightarrow{3} \\
8 \\
\vdots\end{array}$ & $\stackrel{+}{n}$ & $\tilde{o}$ & f̊ & $\bar{\sigma}$ & तె & $\stackrel{\text { fo }}{0}$ & $\stackrel{?}{0}$ & $\stackrel{?}{0}$ & 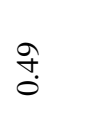 & - & $\underset{\sim}{\stackrel{\infty}{\sim}}$ & $\underset{i}{\stackrel{t}{i}}$ \\
\hline & 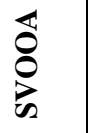 & $\stackrel{\beta}{0}$ & ŏ & 0 & ले & $\vec{\jmath}$ & f. & ले & $\Xi$ & $\stackrel{?}{\rightarrow}$ & $\stackrel{\infty}{\stackrel{0}{0}}$ & $\stackrel{\gtrless}{\stackrel{m}{r}}$ & $\underline{.}$ \\
\hline & 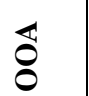 & in & $\stackrel{+}{\infty}$ & $\stackrel{\text { f̊ }}{0}$ & $\stackrel{?}{\longrightarrow}$ & $\stackrel{?}{\stackrel{0}{0}}$ & 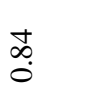 & $\stackrel{\simeq}{\leftrightarrows}$ & $\stackrel{+}{\infty}$ & $\stackrel{\vartheta}{i}$ & $\stackrel{\infty}{\stackrel{\infty}{+}}$ & $\stackrel{\infty}{n}$ & $\overrightarrow{\tilde{n}}$ \\
\hline & 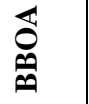 & $\stackrel{\text { Iิ }}{0}$ & 1 & ָָ & $\stackrel{\circ}{\circ}$ & $\overrightarrow{0}$ & $\frac{7}{0}$ & $\stackrel{n}{0}$ & के & ֶֻ. & $n$ & ' & $\stackrel{t}{\stackrel{0}{0}}$ \\
\hline & $\underset{\Xi}{\Xi}$ & તై & 0 & $\overrightarrow{0}$ & $\stackrel{d}{0}$ & $\stackrel{\overbrace{}}{\circ}$ & તై & $\stackrel{m}{0}$ & त़ & $\tilde{n}$ & $\because$ & 0 & 0 \\
\hline \multicolumn{2}{|c|}{ 兽 } & 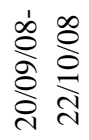 & 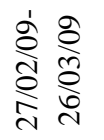 & 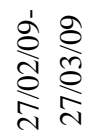 & 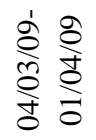 & 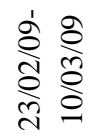 & 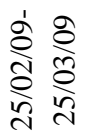 & 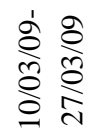 & 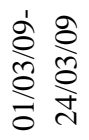 & 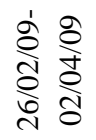 & 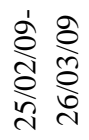 & 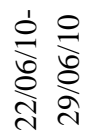 & 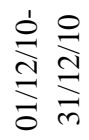 \\
\hline \multirow{2}{*}{ 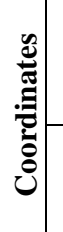 } & 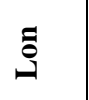 & $\underset{+}{\stackrel{+}{*}}$ & $\begin{array}{l}: \\
\stackrel{0}{0} \\
\dot{a}\end{array}$ & $\stackrel{\infty}{i}$ & $\stackrel{m}{\stackrel{\sim}{d}}$ & $\hat{\mathrm{i}}$ & $\stackrel{\wp}{\stackrel{\sigma}{r}}$ & $\begin{array}{l}\hat{\Omega} \\
\stackrel{\Omega}{I}\end{array}$ & $\stackrel{n}{6}$ & $\frac{n}{m}$ & $\stackrel{+}{\rightarrow}$ & $\hat{\mathrm{a}}$ & 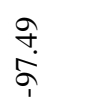 \\
\hline & 离 & $\stackrel{n}{\vec{n}}$ & $\begin{array}{c}m \\
\stackrel{m}{n} \\
m\end{array}$ & $\stackrel{m}{n}$ & $\stackrel{\infty}{\sigma}$ & 衤 & $\frac{\hat{\sigma}}{\dot{n}}$ & 点 & $\begin{array}{l}\infty \\
\dot{\varphi} \\
\dot{\sigma}\end{array}$ & $\begin{array}{l}\text { Dे } \\
\text { in }\end{array}$ & $\Rightarrow$ & 衤 & $\begin{array}{l}\vec{\sigma} \\
\dot{\phi}\end{array}$ \\
\hline $\begin{array}{l} \\
\\
\end{array}$ & 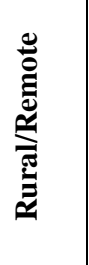 & 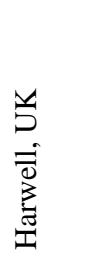 & 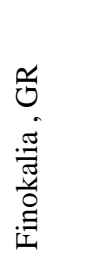 & 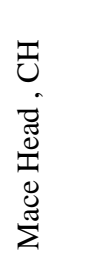 & 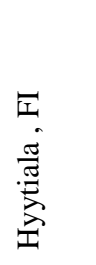 & 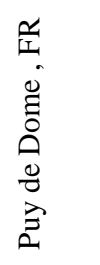 & 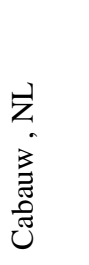 & $\begin{array}{l}\text { Iñ } \\
\text { 劳 } \\
\frac{0}{2}\end{array}$ & 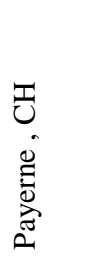 & 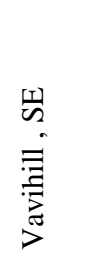 & 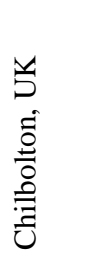 & 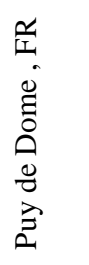 & 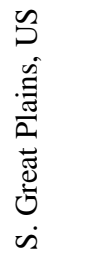 \\
\hline
\end{tabular}




\section{References}

Aiken, A. C., Decarlo, P. F., Kroll, J. H., Worsnop, D. R., Huffman, J. A., Docherty, K. S., Ulbrich, I. M., Mohr, C., Kimmel, J. R., Sueper, D., Sun, Y., Zhang, Q., Trimborn, A., Northway, M., Ziemann, P. J., Canagaratna, M. R., Onasch, T. B., Alfarra, M. R., Prevot, A. S. H., Dommen, J., Duplissy, J., Metzger, A., Baltensperger, U., and Jimenez, J. L.: $\mathrm{O} / \mathrm{C}$ and $\mathrm{OM} / \mathrm{OC}$ ratios of primary, secondary, and ambient organic aerosols with high-resolution time-of-flight aerosol mass spectrometry, Environ. Sci. Technol., 42, 4478-4485, 2008.

Alfarra, M. R., Coe, H., Allan, J. D., Bower, K. N., Boudries, H., Canagaratna, M. R., Jimenez, J. L., Jayne, J. T., Garforth, A. A., Li, S. M., and Worsnop, D. R.: Characterization of urban and rural organic particulate in the lower Fraser valley using two aerodyne aerosol mass spectrometers, Atmo. Environ., 38, 57455758, 2004.

Allan, J. D., Alfarra, M. R., Bower, K. N., Williams, P. I., Gallagher, M. W., Jimenez, J. L., McDonald, A. G., Nemitz, E., Canagaratna, M. R., Jayne, J. T., Coe, H., and Worsnop, D. R.: Quantitative sampling using an Aerodyne aerosol mass spectrometer - 2. Measurements of fine particulate chemical composition in two U.K. cities, J. Geophys. Res. Atmo., 108, doi: 10.1029/2002jd002359, 2003.

Allan, J. D., Alfarra, M. R., Bower, K. N., Coe, H., Jayne, J. T., Worsnop, D. R., Aalto, P. P., Kulmala, M., Hyotylainen, T., Cavalli, F., and Laaksonen, A.: Size and composition measurements of background aerosol and new particle growth in a Finnish forest during QUEST 2 using an Aerodyne Aerosol Mass Spectrometer, Atmos. Chem. Phys., 6, 315-327, 2006.

Bae, M.-S., Schwab, J. J., Zhang, Q., Hogrefe, O., Demerjian, K. L., Weimer, S., Rhoads, K., Orsini, D., Venkatachari, P., and Hopke, P. K.: Interference of organic signals in highly time resolved nitrate measurements by low mass resolution aerosol mass spectrometry, J. Geophys. Res. Atmo., 112, doi: doi: 10.1029/2007jd008614, 2007.

Boudries, H., Canagaratna, M. R., Jayne, J. T., Alfarra, M. R., Allan, J., Bower, K. N., Coe, H., Pryor, S. C., Jimenez, J. L., Brook, J. R., Li, S., and Worsnop, D. R.: Chemical and physical processes controlling the distribution of aerosols in the Lower Fraser Valley, Canada, during the Pacific 2001 field campaign, Atmo. Environ., 38, 5759-5774, 2004.

Carbone, S., Aurela, M., Saarnio, K., Saarikoski, S., Timonen, H., Frey, A., Sueper, D., Ulbrich, I. M., Jimenez, J. L., Kulmala, M., Worsnop, D. R., and Hillamo, R. E.: Wintertime Aerosol Chemistry in Sub-Arctic Urban Air, Aerosol Sci. Technol., 48, 313-323, 2014.

Cottrell, L. D., Griffin, R. J., Jimenez, J. L., Zhang, Q., Ulbrich, I., Ziemba, L. D., Beckman, P. J., Sive, B. C., and Talbot, R. W.: Submicron particles at Thompson Farm during ICARTT measured using aerosol mass spectrometry, J. Geophys. Res. Atmo., 113, doi: doi: 10.1029/2007jd009192, 2008.

Crippa, M., El Haddad, I., Slowik, J. G., DeCarlo, P. F., Mohr, C., Heringa, M. F., Chirico, R., Marchand, N., Sciare, J., Baltensperger, U., and Prevot, A. S. H.: Identification of marine and continental aerosol sources in Paris using high resolution aerosol mass spectrometry, J. Geophys. Res. Atmo., 118, 1950-1963, doi: 10.1002/jgrd.50151, 2013a.

Crippa, M., DeCarlo, P. F., Slowik, J. G., Mohr, C., Heringa, M. F., Chirico, R., Poulain, L., Freutel, F., Sciare, J., Cozic, J., Di Marco, C. F., Elsasser, M., Nicolas, J. B., Marchand, N., Abidi, E., Wiedensohler, A., Drewnick, F., Schneider, J., Borrmann, S., Nemitz, E., Zimmermann, R., Jaffrezo, J. L., 
Prevot, A. S. H., and Baltensperger, U.: Wintertime aerosol chemical composition and source apportionment of the organic fraction in the metropolitan area of Paris, Atmos. Chem. Phys., 13, 961-981, 2013b.

Crippa, M., Canonaco, F., Lanz, V. A., Aijala, M., Allan, J. D., Carbone, S., Capes, G., Ceburnis, D., Dall'Osto, M., Day, D. A., DeCarlo, P. F., Ehn, M., Eriksson, A., Freney, E., Ruiz, L. H., Hillamo, R., Jimenez, J. L., Junninen, H., KiendlerScharr, A., Kortelainen, A. M., Kulmala, M., Laaksonen, A., Mensah, A., Mohr, C., Nemitz, E., O'Dowd, C., Ovadnevaite, J., Pandis, S. N., Petaja, T., Poulain, L., Saarikoski, S., Sellegri, K., Swietlicki, E., Tiitta, P., Worsnop, D. R., Baltensperger, U., and Prevot, A. S. H.: Organic aerosol components derived from 25 AMS data sets across Europe using a consistent ME-2 based source apportionment approach, Atmos. Chem. Phys., 14, 6159-6176, 2014.

Cubison, M. J., Alfarra, M. R., Allan, J., Bower, K. N., Coe, H., McFiggans, G. B., Whitehead, J. D., Williams, P. I., Zhang, Q., Jimenez, J. L., Hopkins, J., and Lee, J.: The characterisation of pollution aerosol in a changing photochemical environment, Atmos. Chem. Phys., 6, 5573-5588, 2006.

Dall'Osto, M., Ceburnis, D., Martucci, G., Bialek, J., Dupuy, R., Jennings, S. G., Berresheim, H., Wenger, J., Healy, R., Facchini, M. C., Rinaldi, M., Giulianelli, L., Finessi, E., Worsnop, D., Ehn, M., Mikkilä, J., Kulmala, M., and O'Dowd, C. D.: Aerosol properties associated with air masses arriving into the North East Atlantic during the 2008 Mace Head EUCAARI intensive observing period: an overview, Atmos. Chem. Phys., 10, 8413-8435, 2010.

de Gouw, J. A., Middlebrook, A. M., Warneke, C., Goldan, P. D., Kuster, W. C., Roberts, J. M., Fehsenfeld, F. C., Worsnop, D. R., Canagaratna, M. R., Pszenny, A. A. P., Keene, W. C., Marchewka, M., Bertman, S. B., and Bates, T. S.: Budget of organic carbon in a polluted atmosphere: Results from the New England Air Quality Study in 2002, J. Geophys. Res. Atmo., 110, doi: doi: 10.1029/2004jd005623, 2005.

DeCarlo, P. F., Kimmel, J. R., Trimborn, A., Northway, M. J., Jayne, J. T., Aiken, A. C., Gonin, M., Fuhrer, K., Horvath, T., Docherty, K. S., Worsnop, D. R., and Jimenez, J. L.: Field-deployable, high-resolution, time-of-flight aerosol mass spectrometer, Anal. Chem., 78, 8281-8289, doi: 10.1021/ac061249n, 2006.

Drewnick, F., Jayne, J. T., Canagaratna, M., Worsnop, D. R., and Demerjian, K. L.: Measurement of ambient aerosol composition during the PMTACS-NY 2001 using an aerosol mass spectrometer. Part II: Chemically speciated mass distributions, Aerosol Sci. Technol., 38, 104-117, 2004.

Dusek, U., Frank, G. P., Hildebrandt, L., Curtius, J., Schneider, J., Walter, S., Chand, D., Drewnick, F., Hings, S., Jung, D., Borrmann, S., and Andreae, M. O.: Size matters more than chemistry for cloud-nucleating ability of aerosol particles, Science, 312, 1375-1378, 2006.

Favez, O., El Haddad, I., Piot, C., Boreave, A., Abidi, E., Marchand, N., Jaffrezo, J. L., Besombes, J. L., Personnaz, M. B., Sciare, J., Wortham, H., George, C., and D'Anna, B.: Inter-comparison of source apportionment models for the estimation of wood burning aerosols during wintertime in an Alpine city (Grenoble, France), Atmos. Chem. Phys., 10, 5295-5314, 2010.

Fountoukis, C., Megaritis, A. G., Skyllakou, K., Charalampidis, P. E., Denier van der Gon, H. A. C., Crippa, M., Prévôt, A. S. H., Freutel, F., Wiedensohler, A., Pilinis, C., and Pandis, S. N.: Simulating the formation of carbonaceous aerosol in a European Megacity (Paris) during the MEGAPOLI summer and winter campaigns, Atmos. Chem. Phys. Discuss., 15, 25547-25582, 2015. 
Freney, E. J., Sellegri, K., Canonaco, F., Boulon, J., Hervo, M., Weigel, R., Pichon, J. M., Colomb, A., Prévôt, A. S. H., and Laj, P.: Seasonal variations in aerosol particle composition at the puy-de-Dôme research station in France, Atmos. Chem. Phys., 11, 13047-13059, 2011.

Ge, X., Setyan, A., Sun, Y., and Zhang, Q.: Primary and secondary organic aerosols in Fresno, California during wintertime: Results from high resolution aerosol mass spectrometry, J. Geophys. Res. Atmo., 117, doi: 10.1029/2012jd018026, 2012.

Gysel, M., Crosier, J., Topping, D. O., Whitehead, J. D., Bower, K. N., Cubison, M. J., Williams, P. I., Flynn, M. J., McFiggans, G. B., and Coe, H.: Closure study between chemical composition and hygroscopic growth of aerosol particles during TORCH2, Atmos. Chem. Phys., 7, 6131-6144, 2007.

Hayes, P. L., Ortega, A. M., Cubison, M. J., Froyd, K. D., Zhao, Y., Cliff, S. S., Hu, W. W., Toohey, D. W., Flynn, J. H., Lefer, B. L., Grossberg, N., Alvarez, S., Rappenglueck, B., Taylor, J. W., Allan, J. D., Holloway, J. S., Gilman, J. B., Kuster, W. C., De Gouw, J. A., Massoli, P., Zhang, X., Liu, J., Weber, R. J., Corrigan, A. L., Russell, L. M., Isaacman, G., Worton, D. R., Kreisberg, N. M., Goldstein, A. H., Thalman, R., Waxman, E. M., Volkamer, R., Lin, Y. H., Surratt, J. D., Kleindienst, T. E., Offenberg, J. H., Dusanter, S., Griffith, S., Stevens, P. S., Brioude, J., Angevine, W. M., and Jimenez, J. L.: Organic aerosol composition and sources in Pasadena, California, during the 2010 CalNex campaign, J. Geophys. Res. Atmo., 118, 9233-9257, doi: 10.1002/jgrd.50530, 2013.

Hildebrandt, L., Engelhart, G. J., Mohr, C., Kostenidou, E., Lanz, V. A., Bougiatioti, A., DeCarlo, P. F., Prevot, A. S. H., Baltensperger, U., Mihalopoulos, N., Donahue, N. M., and Pandis, S. N.: Aged organic aerosol in the Eastern Mediterranean: the Finokalia Aerosol Measurement Experiment-2008, Atmos. Chem. Phys., 10, 4167-4186, 2010.

Hildebrandt, L., Kostenidou, E., Lanz, V. A., Prevot, A. S. H., Baltensperger, U., Mihalopoulos, N., Laaksonen, A., Donahue, N. M., and Pandis, S. N.: Sources and atmospheric processing of organic aerosol in the Mediterranean: insights from aerosol mass spectrometer factor analysis, Atmos. Chem. Phys., 11, 2011.

Hock, N., Schneider, J., Borrmann, S., Roempp, A., Moortgat, G., Franze, T., Schauer, C., Poeschl, U., Plass-Duelmer, C., and Berresheim, H.: Rural continental aerosol properties and processes observed during the Hohenpeissenberg Aerosol Characterization Experiment (HAZE2002), Atmos. Chem. Phys., 8, 603-623, 2008.

Holzinger, R., Millet, D. B., Williams, B., Lee, A., Kreisberg, N., Hering, S. V., Jimenez, J., Allan, J. D., Worsnop, D. R., and Goldstein, A. H.: Emission, oxidation, and secondary organic aerosol formation of volatile organic compounds as observed at Chebogue Point, Nova Scotia, J. Geophys. Res. Atmo., 112, doi: 10.1029/2006jd007599, 2007.

Jimenez, J. L., Canagaratna, M. R., Donahue, N. M., Prevot, A. S. H., Zhang, Q., Kroll, J. H., DeCarlo, P. F., Allan, J. D., Coe, H., Ng, N. L., Aiken, A. C., Docherty, K. S., Ulbrich, I. M., Grieshop, A. P., Robinson, A. L., Duplissy, J., Smith, J. D., Wilson, K. R., Lanz, V. A., Hueglin, C., Sun, Y. L., Tian, J., Laaksonen, A., Raatikainen, T., Rautiainen, J., Vaattovaara, P., Ehn, M., Kulmala, M., Tomlinson, J. M., Collins, D. R., Cubison, M. J., Dunlea, E. J., Huffman, J. A., Onasch, T. B., Alfarra, M. R., Williams, P. I., Bower, K., Kondo, Y., Schneider, J., Drewnick, F., Borrmann, S., Weimer, S., Demerjian, 
K., Salcedo, D., Cottrell, L., Griffin, R., Takami, A., Miyoshi, T., Hatakeyama, S., Shimono, A., Sun, J. Y., Zhang, Y. M., Dzepina, K., Kimmel, J. R., Sueper, D., Jayne, J. T., Herndon, S. C., Trimborn, A. M., Williams, L. R., Wood, E. C., Middlebrook, A. M., Kolb, C. E., Baltensperger, U., and Worsnop, D. R.: Evolution of organic aerosols in the atmosphere, Science, 326, 1525-1529, 2009.

Lanz, V. A., Alfarra, M. R., Baltensperger, U., Buchmann, B., Hueglin, C., and Prevot, A. S. H.: Source apportionment of submicron organic aerosols at an urban site by factor analytical modelling of aerosol mass spectra, Atmos. Chem. Phys., 7, 1503-1522, 2007.

Lanz, V. A., Alfarra, M. R., Baltensperger, U., Buchmann, B., Hueglin, C., Szidat, S., Wehrli, M. N., Wacker, L., Weimer, S., Caseiro, A., Puxbaum, H., and Prevot, A. S. H.: Source attribution of submicron organic aerosols during wintertime inversions by advanced factor analysis of aerosol mass spectra, Environ. Sci. Technol., 42, 214-220, 2008.

Lanz, V. A., Prevot, A. S. H., Alfarra, M. R., Weimer, S., Mohr, C., DeCarlo, P. F., Gianini, M. F. D., Hueglin, C., Schneider, J., Favez, O., D'Anna, B., George, C., and Baltensperger, U.: Characterization of aerosol chemical composition with aerosol mass spectrometry in Central Europe: an overview, Atmos. Chem. Phys., 10, 2010.

Mensah, A. A., Holzinger, R., Otjes, R., Trimborn, A., Mentel, T. F., ten Brink, H., Henzing, B., and Kiendler-Scharr, A.: Aerosol chemical composition at Cabauw, The Netherlands as observed in two intensive periods in May 2008 and March 2009, Atmos. Chem. Phys., 12, 4723-4742, 2012.

Mohr, C., DeCarlo, P. F., Heringa, M. F., Chirico, R., Slowik, J. G., Richter, R., Reche, C., Alastuey, A., Querol, X., Seco, R., Penuelas, J., Jimenez, J. L., Crippa, M., Zimmermann, R., Baltensperger, U., and Prevot, A. S. H.: Identification and quantification of organic aerosol from cooking and other sources in Barcelona using aerosol mass spectrometer data, Atmos. Chem. Phys., 12, 1649-1665, 2012.

Nemitz, E., Jimenez, J. L., Huffman, J. A., Ulbrich, I. M., Canagaratna, M. R., Worsnop, D. R., and Guenther, A. B.: An eddy-covariance system for the measurement of surface/atmosphere exchange fluxes of submicron aerosol chemical species - First application above an urban area, Aerosol Sci. Technol., 42, 636-657, 2008.

Parworth, C., Fast, J., Mei, F., Shippert, T., Sivaraman, C., Tilp, A., Watson, T., and Zhang, Q.: Long-term measurements of submicrometer aerosol chemistry at the Southern Great Plains (SGP) using an Aerosol Chemical Speciation Monitor (ACSM), Atmo. Environ., 106, 43-55, 2015.

Poulain, L., Spindler, G., Birmili, W., Plass-Dülmer, C., Wiedensohler, A., and Herrmann, H.: Seasonal and diurnal variations of particulate nitrate and organic matter at the IfT research station Melpitz, Atmos. Chem. Phys., 11, 1257912599, 2011.

Richardson, M. S., DeMott, P. J., Kreidenweis, S. M., Cziczo, D. J., Dunlea, E. J., Jimenez, J. L., Thomson, D. S., Ashbaugh, L. L., Borys, R. D., Westphal, D. L., Casuccio, G. S., and Lersch, T. L.: Measurements of heterogeneous ice nuclei in the western United States in springtime and their relation to aerosol characteristics, J. Geophys. Res. Atmo., 112, doi: 10.1029/2006jd007500, 2007.

Saarikoski, S., Carbone, S., Decesari, S., Giulianelli, L., Angelini, F., Canagaratna, M., Ng, N. L., Trimborn, A., Facchini, M. C., Fuzzi, S., Hillamo, R., and 
Worsnop, D.: Chemical characterization of springtime submicrometer aerosol in Po Valley, Italy, Atmos. Chem. Phys., 12, 8401-8421, 2012.

Salcedo, D., Onasch, T. B., Dzepina, K., Canagaratna, M. R., Zhang, Q., Huffman, J. A., DeCarlo, P. F., Jayne, J. T., Mortimer, P., Worsnop, D. R., Kolb, C. E., Johnson, K. S., Zuberi, B., Marr, L. C., Volkamer, R., Molina, L. T., Molina, M. J., Cardenas, B., Bernabe, R. M., Marquez, C., Gaffney, J. S., Marley, N. A., Laskin, A., Shutthanandan, V., Xie, Y., Brune, W., Lesher, R., Shirley, T., and Jimenez, J. L.: Characterization of ambient aerosols in Mexico City during the MCMA-2003 campaign with Aerosol Mass Spectrometry: results from the CENICA Supersite, Atmos. Chem. Phys., 6, 925-946, 2006.

Setyan, A., Zhang, Q., Merkel, M., Knighton, W. B., Sun, Y., Song, C., Shilling, J. E., Onasch, T. B., Herndon, S. C., Worsnop, D. R., Fast, J. D., Zaveri, R. A., Berg, L. K., Wiedensohler, A., Flowers, B. A., Dubey, M. K., and Subramanian, R.: Submicron particles influenced by mixed biogenic and anthropogenic emissions: high-resolution aerosol mass spectrometry results from the Carbonaceous Aerosols and Radiative Effects Study (CARES), Atmos. Chem. Phys. Discuss., 12, 5601-5658, 2012.

Sun, J., Zhang, Q., Canagaratna, M. R., Zhang, Y., Ng, N. L., Sun, Y., Jayne, J. T., Zhang, X., Zhang, X., and Worsnop, D. R.: Highly time- and size-resolved characterization of submicron aerosol particles in Beijing using an Aerodyne Aerosol Mass Spectrometer, Atmo. Environ., 44, 131-140, 2010.

Sun, Y. L., Zhang, Q., Schwab, J. J., Demerjian, K. L., Chen, W. N., Bae, M. S., Hung, H. M., Hogrefe, O., Frank, B., Rattigan, O. V., and Lin, Y. C.: Characterization of the sources and processes of organic and inorganic aerosols in New York city with a high-resolution time-of-flight aerosol mass apectrometer, Atmos. Chem. Phys., 11, 1581-1602, 10.5194/acp-11-1581-2011, 2011.

Takami, A., Miyoshi, T., Shimono, A., and Hatakeyama, S.: Chemical composition of fine aerosol measured by AMS at Fukue Island, Japan during APEX period, Atmo. Environ., 39, 4913-4924, 2005.

Takegawa, N., Miyazaki, Y., Kondo, Y., Komazaki, Y., Miyakawa, T., Jimenez, J. L., Jayne, J. T., Worsnop, D. R., Allan, J. D., and Weber, R. J.: Characterization of an Aerodyne Aerosol Mass Spectrometer (AMS): Intercomparison with other aerosol instruments, Aerosol Sci. Technol., 39, 760-770, 2005.

Takegawa, N., Miyakawa, T., Kondo, Y., Jimenez, J. L., Zhang, Q., Worsnop, D. R., and Fukuda, M.: Seasonal and diurnal variations of submicron organic aerosol in Tokyo observed using the Aerodyne aerosol mass spectrometer, J. Geophys. Res. Atmo., 111, doi: 10.1029/2005jd006515, 2006.

Timonen, H., Carbone, S., Aurela, M., Saarnio, K., Saarikoski, S., Ng, N. L., Canagaratna, M. R., Kulmala, M., Kerminen, V.-M., Worsnop, D. R., and Hillamo, R.: Characteristics, sources and water-solubility of ambient submicron organic aerosol in springtime in Helsinki, Finland, Journal of Aerosol Science, 56, 61-77, 2013.

Topping, D., Coe, H., McFiggans, G., Burgess, R., Allan, J., Alfarra, M. R., Bower, K., Choularton, T. W., Decesari, S., and Facchini, M. C.: Aerosol chemical characteristics from sampling conducted on the Island of Jeju, Korea during ACE Asia, Atmo. Environ., 38, 2111-2123, 2004.

Tsimpidi, A. P., Karydis, V. A., Zavala, M., Lei, W., Molina, L., Ulbrich, I. M., Jimenez, J. L., and Pandis, S. N.: Evaluation of the volatility basis-set approach 
for the simulation of organic aerosol formation in the Mexico City metropolitan area, Atmos. Chem. Phys., 10, 525-546, 2010.

Tsimpidi, A. P., Karydis, V. A., Zavala, M., Lei, W., Bei, N., Molina, L., and Pandis, S. N.: Sources and production of organic aerosol in Mexico City: insights from the combination of a chemical transport model (PMCAMx-2008) and measurements during MILAGRO, Atmos. Chem. Phys., 11, 5153-5168, 2011.

Vester, B. P., Ebert, M., Barnert, E. B., Schneider, J., Kandler, K., Schuetz, L., and Weinbruch, S.: Composition and mixing state of the urban background aerosol in the Rhein-Main area (Germany), Atmo. Environ., 41, 6102-6115, 2007.

Zhang, Q., Stanier, C. O., Canagaratna, M. R., Jayne, J. T., Worsnop, D. R., Pandis, S. N., and Jimenez, J. L.: Insights into the chemistry of new particle formation and growth events in Pittsburgh based on aerosol mass spectrometry, Environ. Sci. Technol., 38, 4797-4809, 2004.

Zhang, Q., Jimenez, J. L., Canagaratna, M. R., Allan, J. D., Coe, H., Ulbrich, I., Alfarra, M. R., Takami, A., Middlebrook, A. M., Sun, Y. L., Dzepina, K., Dunlea, E., Docherty, K., DeCarlo, P. F., Salcedo, D., Onasch, T., Jayne, J. T., Miyoshi, T., Shimono, A., Hatakeyama, S., Takegawa, N., Kondo, Y., Schneider, J., Drewnick, F., Borrmann, S., Weimer, S., Demerjian, K., Williams, P., Bower, K., Bahreini, R., Cottrell, L., Griffin, R. J., Rautiainen, J., Sun, J. Y., Zhang, Y. M., and Worsnop, D. R.: Ubiquity and dominance of oxygenated species in organic aerosols in anthropogenically-influenced Northern Hemisphere midlatitudes, Geophys. Res. Lett., 34, doi: 10.1029/2007g1029979, 2007. 